\title{
Novel Configurations of Slit Tubular Soft Robotic Actuators and Sensors made with Ionic Polymer Metal Composites (IPMCs)
}

\author{
Ehsan Tabatabaie S and Mohsen Shahinpoor* \\ Department of Mechanical Engineering, University of Maine, USA
}

Submission: May 20, 2018; Published: July 13, 2018

*Corresponding author: Ajay Kumar Choubey, Assistant Professor, Sagar Institute of Research \& Technology, Bhopal, India, Email: a1ja1y@yahoo.co.in

\begin{abstract}
Reported is a new family of ionic polymer metal composites (IPMCs) actuators, energy harvesters and sensors in slit cylindrical/tubular configurations. The slit IPMC cylindrical/tubular elements are made of bending IPMC slit elements distributed symmetrically around a cylindrical mantle. The slit bending elements around the cylindrical mantle may be either straight and/or parallel with the longitudinal axis of the cylinder for linear actuation and sensing in terms of the length of the entire cylinder or may be helically wound around the cylindrical mantle to produce a combination of linear and torsional actuations and even bending for the slit cylindrical tube. This paper further introduces some models and special cases of linear IPMC actuators and sensors in slit cylindrical/tubular form. Experimental results relating the bending deformation to the axial deformations of slit cylinders are also presented. Of particular interest is the emergence of undulating linear actuation and sensing by slit IPMCs and their combination with twisting and bending to create 3-D soft robotic manipulation and undulation like in an elephant trunk.
\end{abstract}

Keywords: Novel Configurations; Slit Tubular; Soft Robotic Actuators; Sensors;Ionic Polymer; Metal Composites

Abbreviations: IPMCs: Ionic Polymer Metal Composites; EAPs: Electro Active Polymers

\section{Introduction}

Ionic polymer-metal composites (IPMCs) are synthetic nanocomposite materials. They belong to the family of electro active polymers (EAPs). IPMCs deform (actuation mode) in an imposed small electric field (few $\mathrm{kV} / \mathrm{m}$ ). Also, they generate electrical fields (sensing/energy harvesting mode) upon physical deformation or via environmental dynamics, like wind or ocean waves (few $\mathrm{mVs}$ for small samples, $10 \mathrm{~mm} \times 40 \mathrm{~mm} \times 0.2 \mathrm{~mm}$ ). They work both in air and polar liquids such as water and blood. In both cases the conjugated cations need to be mobile enough to migrate towards the cathode when an electric field is imposed on them. This mobility is generally triggered by the osmotic pressure created by the migration of hydrated cations. The mobility and reconfiguration of cations can also be initiated if the IPMCs are in or under water. To maintain consistency in actuation and sensing the IPMCs may be encapsulated with thin silicone rubber sheath, by spraying them with silicone spray to maintain a uniform humidity during actuation, energy harvesting and sensing operations.

An early coverage and review of IPMCs were first published in 1998 by Shahinpoor, Bar-Cohen, Xue, Simpson and Smith [1,2]. However, the original idea of ionic polymer actuators and sensors goes back to 1992-93time frames and the pioneering work of
Osada, Oguro, Kawami, Asaka, Takenaka and Shahinpoor [3-14]. IPMCs are composed of an ionic polymer like Nafion $\AA$ or Flemion $\AA$ whose surfaces are chemically plated or physically coated with conductors like platinum or gold. The electrodynamics of charge migration and generation in these materials are governed by the Poisson-Nernst-Planck field equations [15-24]. These materials display artificial muscle behavior under an applied voltage or electric field. For example an applied voltages of 1 to 4 volts for a typical sample of the size $10 \mathrm{~mm} \times 40 \mathrm{~mm} \times 0.2 \mathrm{~mm}$ typically produces spectacular bending (Figure 1).

Ions migration and redistribution, due to the imposed voltage across a strip of IPMC, result in all kinds of deformations. If the plated electrodes are arranged in a non-symmetric configuration, the imposed voltage can induce many kinds of deformations such as twisting, rolling, turning, twirling, whirling and non-symmetric bending deformation. Alternatively, if such deformations are physically applied to an IPMC strip they generate a voltage signal (few millivolts for typical small samples) as sensors and energy harvesters [25-27].

Figure1a \& 1bdepict various deformation configurations of IPMC strips depending on the placement configuration of the 


\section{Robotics \& Automation Engineering Journal}

electrodes chemically plated on the IPMCs. Such twisting/bending deformations of IPMCs (Figure 1b) may be generated by placing the electrodes on the IPMC strip in eccentric and non-symmetrical configurations. These materials generate a force density of about 40 in a cantilever configuration for sizes around $5 \mathrm{~mm} \times 30 \mathrm{~mm} \times$ $0.2 \mathrm{~mm}$, meaning they can generate a tip blocking force of almost
40 times their own weight in a cantilever mode[12,23]. In other words if the weight of a cantilever is about $0.06 \mathrm{gmf}$, based on a density of $2 \mathrm{gm} / \mathrm{cm}^{3}$, the sample can produce a tip blocking force of about 2.4 gmf. Figure 2 displays the actuation and sensing mechanisms in cantilever strips of IPMCs in a graphical manner.

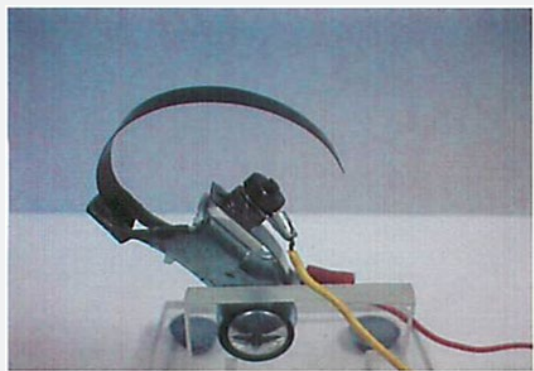

(a)

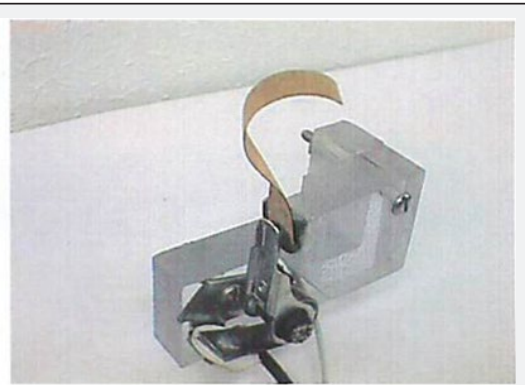

(b)

Figure 1: (a) IPMC strip spectacular bending under 4 volts,

(b) IPMC strip bending \& twisting due to eccentric placement of electrodes.

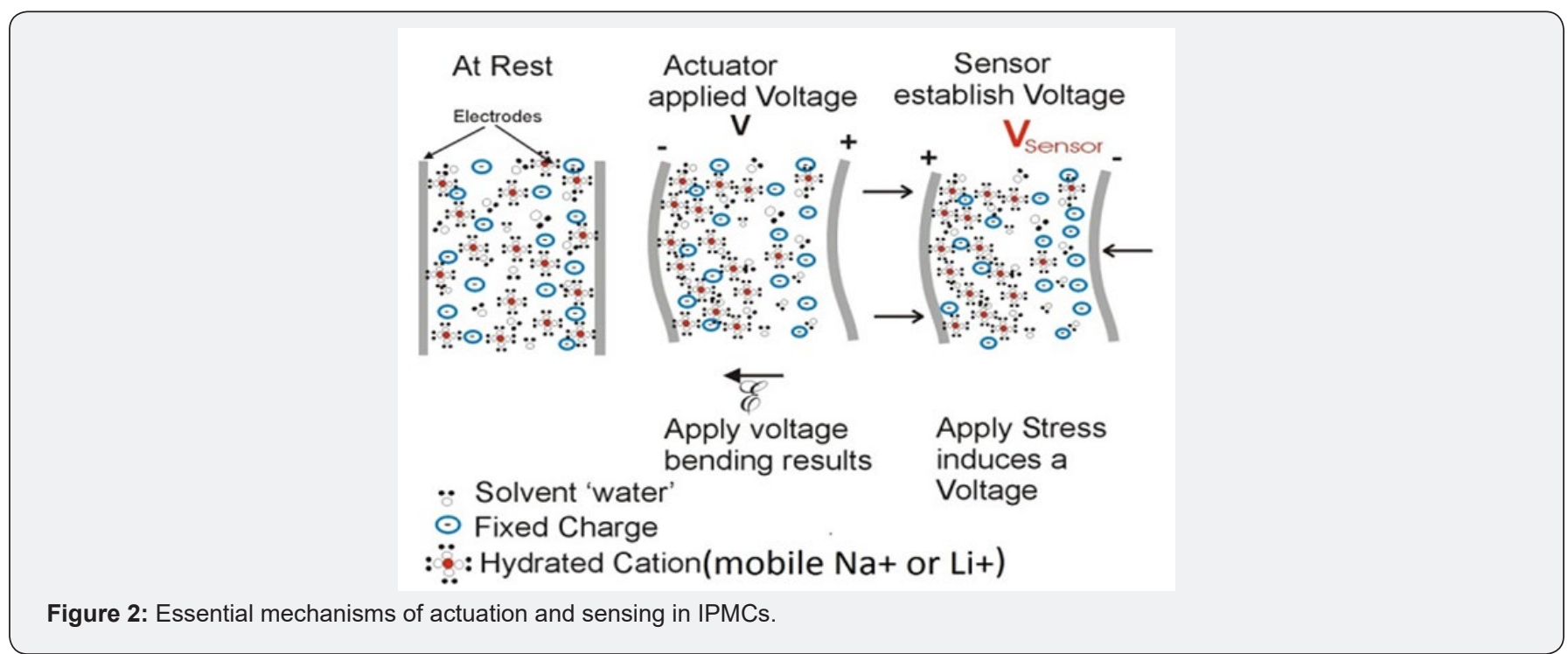

The essential mechanism for both actuation and sensing/ energy harvesting capabilities of IPMCs is the migration of hydrated cations $(\mathrm{Na}+, \mathrm{Li}+)$, which are loosely adjoined to the underlying molecular network with anions, towards the cathode electrode and away from the anode electrode due to either an imposed electric field (actuation) or an imposed deformation field (sensing/energy harvesting). The cations are generally attached to a number of water molecules (hydration number) which is about 4 for $\mathrm{Na}+$ and 6 for Li+. These deformations are considered to be related to contraction due to depletion of cations from the anode side and osmotic pressure or tension due to arrival of hydrated cations on the cathode electrodes side due to Poisson-NernstPlanck phenomena $[23,24]$. It is expected that for a typical sample IPMCs in actuation, sensing and energy harvesting modes there exists a broad bandwidth (kilo HZ and beyond) for their actuation, energy harvesting and sensing response to an imposed electric field or deformation field. The migration of hydrated cations depends on the structure of the base material (DuPont Nafion ${ }^{\circledR}$, Asahi Glass Flemion ${ }^{\circledR}$ ) and how uniform the electrodes are plated on the base material.

In the next section, a brief mathematical model for the actuation and sensing of IPMC is presented.

\section{IPMC Actuation and Sensing Modeling}

de Gennes and coworkers [15] presented the first phenomenological theory for sensing and actuation in ionic polymer metal composites. Asaka et al. [16] discussed the bending of polyelectrolyte membrane-platinum composites by electric stimuli and presented a theory on actuation mechanisms in IPMC by considering the electro-osmotic drag term in transport equations. Figure 2 clearly displays the mechanisms of actuation and sensing of IPMCs. 


\section{Robotics \& Automation Engineering Journal}

Let us now summarize the underlying principle of the Ionic polymeric nanocomposites actuation and sensing capabilities, which can be described by the standard Onsager formulation using linear irreversible thermodynamics. When static conditions are imposed, a simple description of mechanoelectric effect is possible based upon two forms of transport: ion transport (with a current density, $\underset{\sim}{J}$, normal to the material) and solvent transport (with a flux, $Q$, we can assume that this term is water flux). The conjugate forces include the electric field, $\underset{\sim}{E}$ and the pressure gradient $\nabla p(x, y, z, t)$. The resulting equation has the concise form of

$$
\begin{aligned}
& \underset{\sim}{J}(x, y, z, t)=\sigma \underset{\sim}{E}(x, y, z, t)-L_{12} \underset{\sim}{\nabla} p(x, y, z, t) \\
& \underset{\sim}{Q}(x, y, z, t)=L_{21} \underset{\sim}{E}(x, y, z, t)-K \underset{\sim}{\nabla} p(x, y, z, t)
\end{aligned}
$$

Where, $\sigma$ and $K$ are the material electric conductance and the Darcy permeability coefficient, respectively. A cross coefficient (Onsager's coefficient) is usually symmetric or $L=L_{12}=L_{21}$. The simplicity of the above equations provides a compact view of the underlying principles of actuation, transduction and sensing of the ionic polymer nanocomposites. When we measure the direct effect (actuation mode) we work (ideally) with electrodes which are impermeable to ion species flux, and thus we have $Q=0$. This simplifies the equations to:

$$
\underset{\sim}{Q}(x, y, z, t)=L_{21} \underset{\sim}{E}(x, y, z, t)-K \underset{\sim}{\nabla} p(x, y, z, t)
$$

This $\underset{\sim}{\nabla} p(x, y, z, t)$ will, in turn, induce a curvature $\underset{\sim}{k}$ proportional to $\underset{\sim}{\nabla}(x, y, z, t)$. The relationships between the curvature $k$ and pressure gradient $\nabla p(x, y, z, t)$ are fully derived and described in de Gennes, Okumura, Shahinpoor and Kim [15].

Note that $\left(1 / \rho_{c}\right)=M(E) / Y I$, where $M(E)$ is the local induced bending moment and is a function of the imposed electric field E, Y is the Young's modulus (elastic stiffness) of the strip which is a function of the hydration $\mathrm{H}$ of the ionic polymer metal nanocomposite and I is the moment of inertia of the strip. Note that locally $\mathrm{M}(\mathrm{E})$ is related to the pressure gradient such that in a simplified scalar format [15]:

$$
\underset{\sim}{\nabla} p(x, y, z, t)=\left(2 P / t^{*}\right)=(M / I)=Y / \rho_{c}=Y \underset{\sim}{k}
$$

Note that vectorially the curvature $k_{E}$ is related to the imposed electric field $E$ by $\underset{\sim}{K}=(L / K Y) \underset{\sim}{E} \underset{\sim}{\sim}$. Based on this simplified model the tip bending deflection $\delta_{\max }$ of an IPMC strip of length $l_{g}$ can be shown to be almost linearly related to the imposed electric field. The experimental deformation characteristics of IPMCs are clearly consistent with the above predictions obtained by the above linear irreversible thermodynamics formulation which is also consistent with the above equation in the steady state conditions. Note also that the value of the Onsager coefficient $\mathrm{L}$ has been estimated to be of the order of $10^{-8} \mathrm{~m}^{2} / \mathrm{V}$-s [20-24]. Other parameters have been experimentally measured to be $\mathrm{K} \sim 10^{-}$ ${ }^{18} \mathrm{~m}^{2} / \mathrm{CP}, \sigma \sim 1 \mathrm{~A} / \mathrm{mV}$ or $\mathrm{S} / \mathrm{m}$. On the other hand one may consider charge transport modeling of actuation and sensing. The reader is referred to Bahramzadeh and Shahinpoor [20-24] and Shahinpoor [22-24] for such Poisson-Nernst-Planck equations. Similar to poly (ethylene-co-methacrylic acid) metal composite, member of the electroactive polymer can be introduced as biomimetic soft robotic polymer [28-32].

\section{Some Experimental Results}

Figures $3(\mathrm{a}, \mathrm{b} \& \mathrm{c})$ represent experimental results for deformation (tip displacement and curvature) of a small strip of slit IPMC ( $40 \mathrm{~mm} \times 12 \mathrm{~mm} \times 0.2 \mathrm{~mm})$ with simple-supported ends in a small electric field (under 5 Volts $/ 0.2 \mathrm{~mm}$ ) in horizontal and vertical directions. For each graph the bestfittedcurve is constructed to demonstrate the consistency in material response.

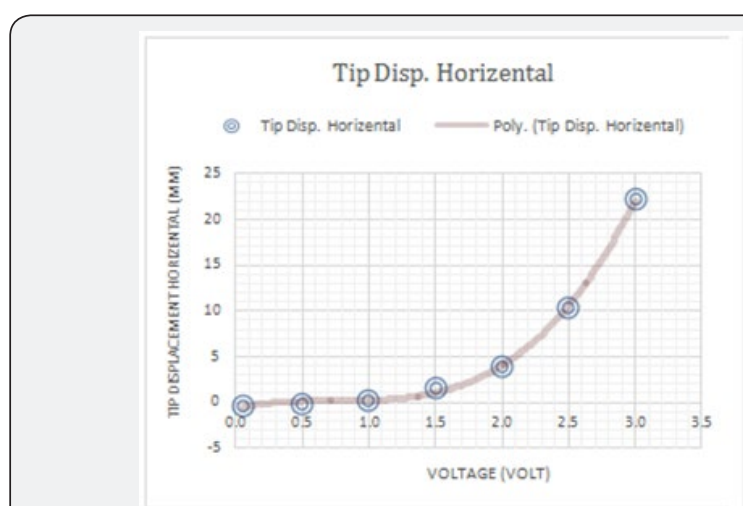

Figure 3a: Horizontal Tip Displacement, Polynomial O3 curve fitted.

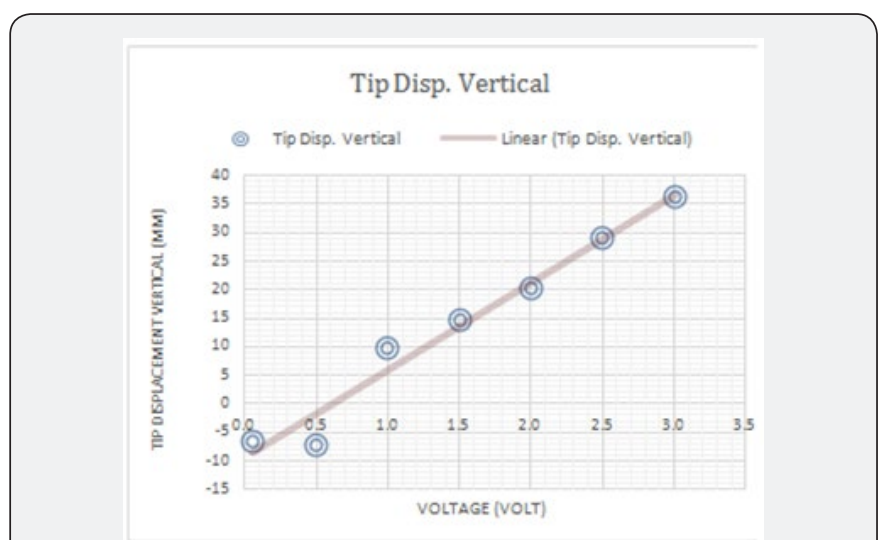

Figure 3b: Vertical Tip Displacement, Linear curve fitted.

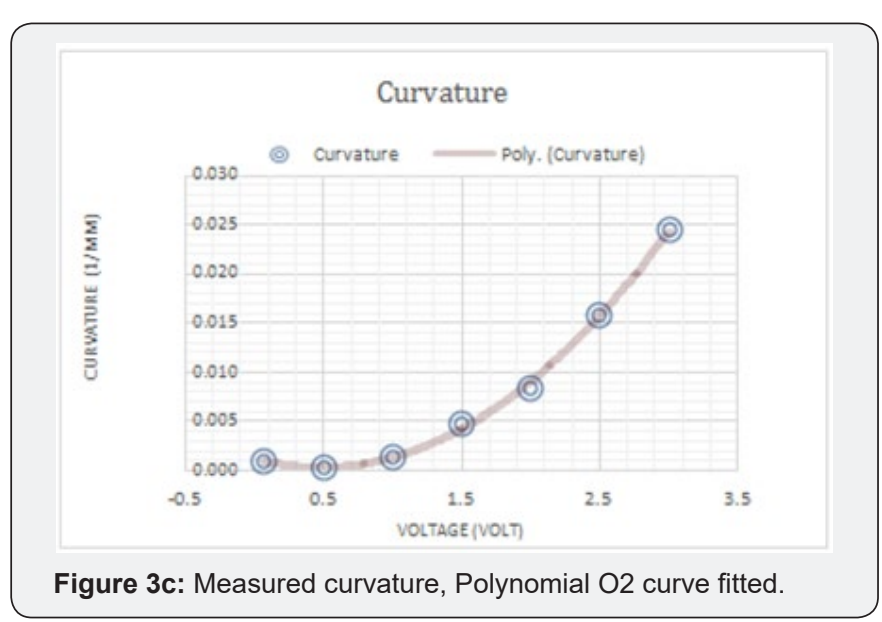




\section{Robotics \& Automation Engineering Journal}

Another set of experimental results were obtained and plotted in connection with slit IPMC strips with built-in ends to recapture the tip displacements in vertical and horizontal configurations, as well as curvature. The results for the second test series are presented in Figure 4(a,b\&c).
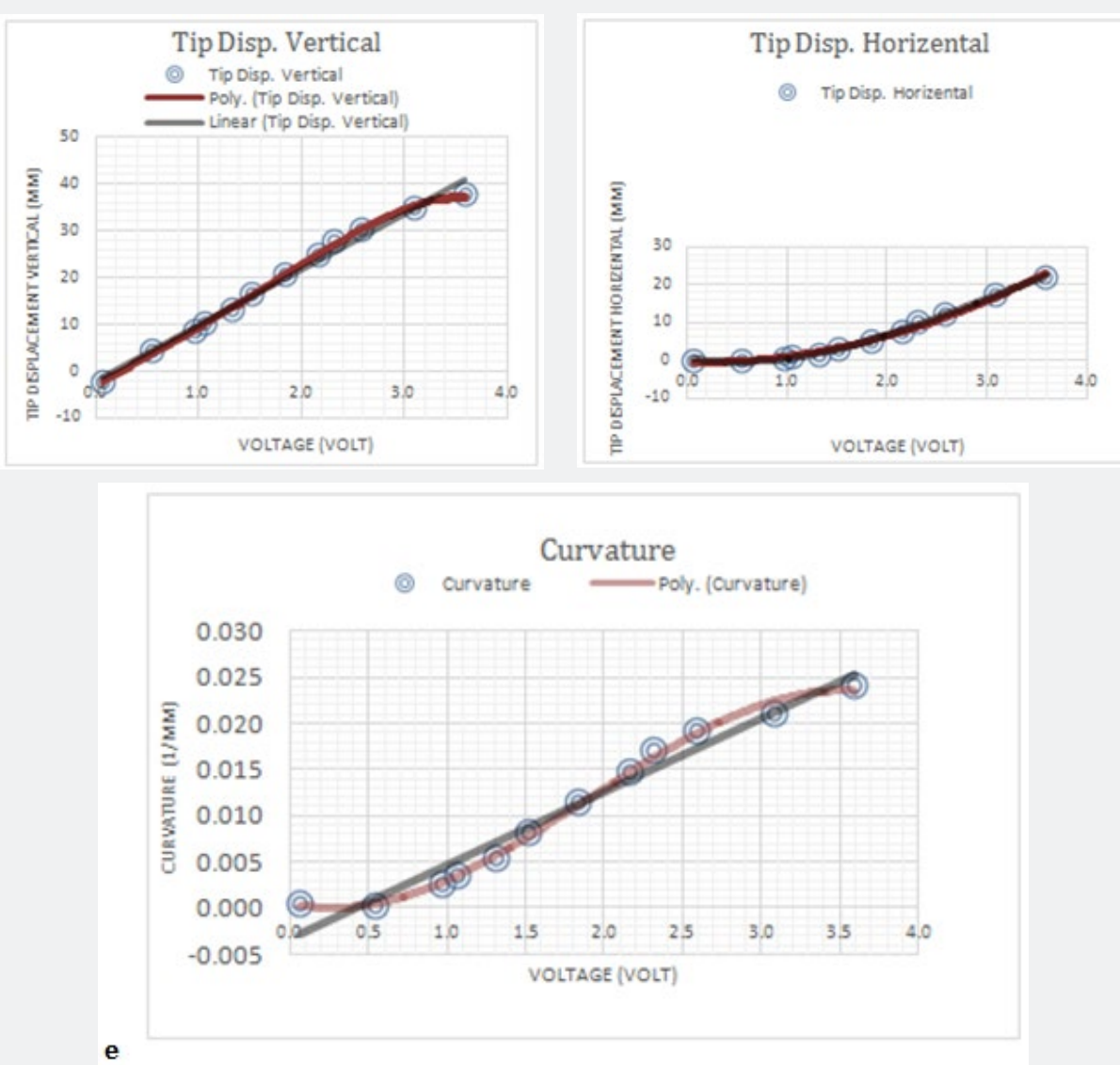

Figure 4: (a) O-2 Polynomial-Fitted Horizontal Tip Displacement,

(b) Vertical Tip Displacement,

(c) Measured Curvature, versus the imposed electric field.

Figure 5(a\&b) represents sensing and energy harvesting sensing of IPMCs are of interest to soft robotic researchers. characteristics of IPMCs [12,23]. Simultaneous actuation and
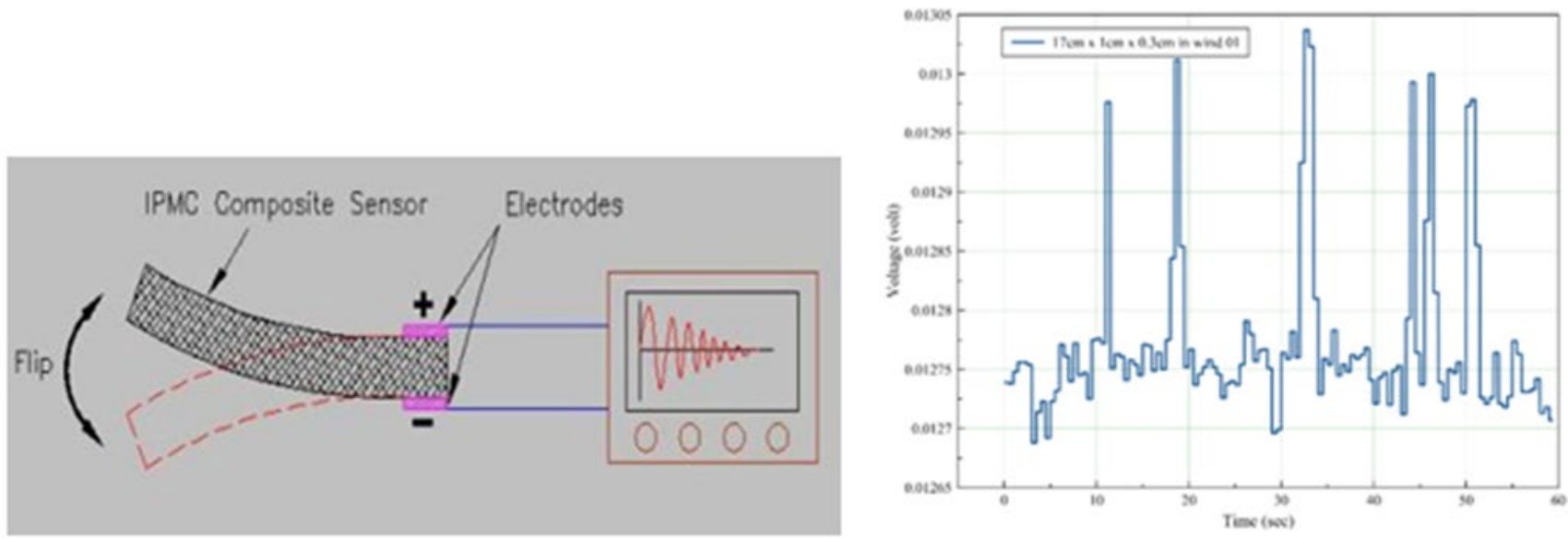

Figure 5: IPMC energy harvesting/sensing configuration. (a) output voltage for an IPMC sample $(17 \mathrm{~cm} \times 1 \mathrm{~cm} \times 0.3 \mathrm{~mm})$ in a cantilever mode oscillating in an arbitrary manner. (b) Novel IPMCs Tubular Configurations as Linear Actuators/Sensors eld. 


\section{Robotics \& Automation Engineering Journal}

Note that IPMCs havea broad range of applicationsin soft biomimetic robotic actuation, energy harvesting and sensing [23-24]. In this section some novel configurations for IPMC actuators and sensors/energy harvesterswill be introduced. Based on the reported test results in Figure 3\&4 for large deformation and actuation of IPMC materials, it is expected these novel configurations will be practically useful for engineering applications.Asimpledescription of actuation in this type of material is based on cationic migration towards the cathode to expand the cathode side of IPMC by osmotic pressure and contract the anode side of the IPMC resulting in bending towards the anode side as depicted in Figure 2.

In other cases and configurations, the IPMC sample can be considered as a non-slit cylinder/tube such that it can act like a linear actuator and generate an axial force (Figure 6).
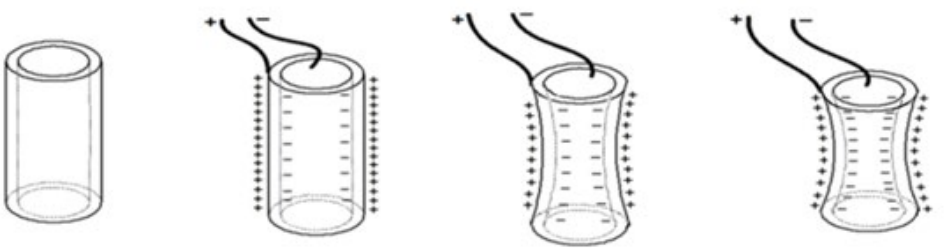

(a)

(b)

(c)

(d)

Figure 6: The principle of linear actuation by IPMC cylinder,
(a) No voltage,
(b) Small voltage
(c) Moderate voltage
(d) High voltage

In the sensing mode, the axial forces on both ends force the cylinder to shrink and thus, as a result, the material will accommodate the deformation. This accommodation can be inward or outward. The pressure gradient due to this accommodation will generate the expected signal to sense the displacement [12]. Regarding the buckling instability under axial loading, the non-slit cylindrical/tubular structures will have initial slight curvature outward or inward to minimize buckling instabilities. In addition, from actuation point of view, an applied electric field causes the non-slit cylinder/tube to contract/expand axially/laterally as shown in Figure 6, based on the voltage polarity. This applied field will gradually deform the cylinder to become more like a sphere (Figure 6d).

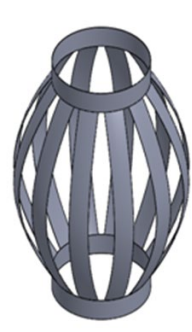

(a)

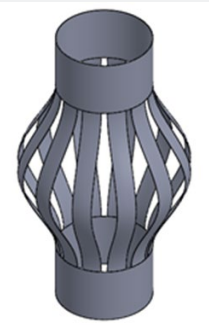

(b)

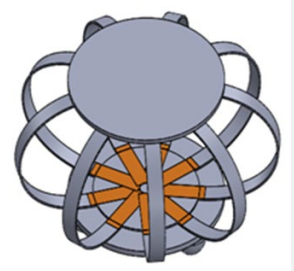

(c)

Figure 7: (a) Slit Tubular form of IPMC actuator with hinge - hinge endings.

(b) Tubular form of IPMC actuator with vertical clamped-clamped endings.

(c) Tubular form of IPMC actuator with horizontal clamp-clamp endings. 


\section{Robotics \& Automation Engineering Journal}

Another case will be using IPMC strips around a cylindrical/ tubular object or a slit tubular IPMC. Some of the configurations of slit IPMC strips around a cylindrical/tubular mantle are shown below in Figure $7(a, b \& c)$.

In the slit tubular configurations in Figure 7, slit IPMC cylindrical strips experience two major forms of boundary condition for this type of the actuator/sensor family (Hinged in Figure 8a and clamped in Figure 7(b\&c). Other form of boundary condition will provide more complex manipulation and maneuver possibilities that will be discussed later. These concept also are likely to be used for applications in pumps/micro-pumps in which the pushing or taking in a fluid can be performed by bending/ deformation of the slit strips in which an inner housing should accompany the assembly. In other word the volume change due to the movement of the IPMCs will initiate the pumping action.

Regarding the sensing mode, whenfor the slit IPMC strips are in a hinge-hinge or simply-supported configuration, there will be less force requirement for the desired displacement andit further results in generatinghigher output voltage. Further, during sensing action the effective length of the IPMC strip is longer. Figure 8, demonstrate the curvature variations related to this formation such that the red regions are in compression and green regions are in tension and the green parts are almost straight.

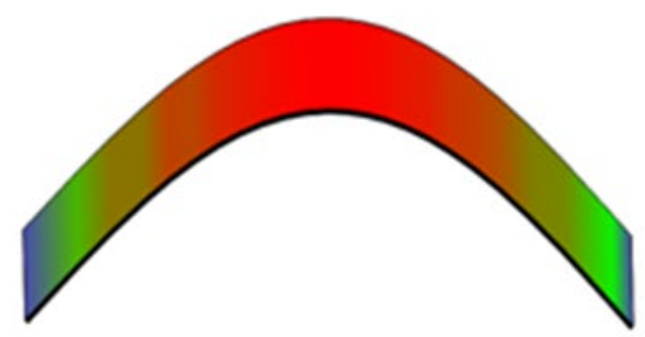

(a)

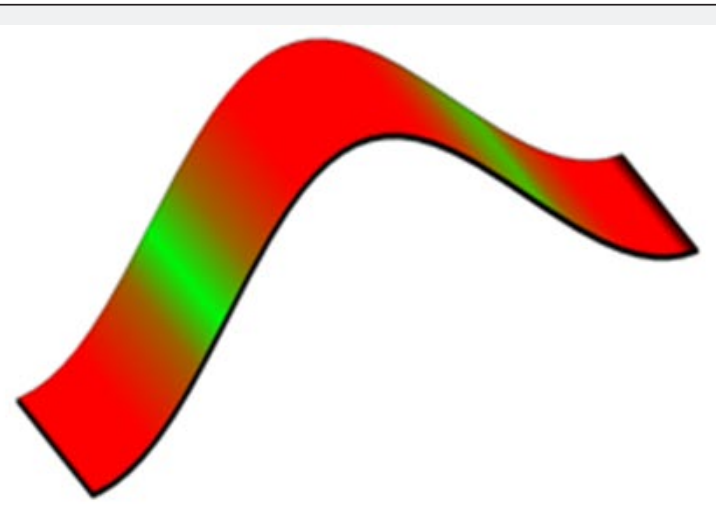

(b)

Figure 8: (a) Variation of curvature of a slit IPMC strip with simply-supported ends and

(b) Variation of curvature of a slit IPMC strip with clamped-clamped ends.

It is also reasonable to note that due to having longer effective length, the hinged-hinged model will have a larger force capacity in actuation. The model with clamped ending will have a shorter effective length to generate output signal and requires a higher level of voltage to operate.

Figure 9 depict a simple form of slit IPMC cylinders in straight simple pinned ending format (Figure 10).

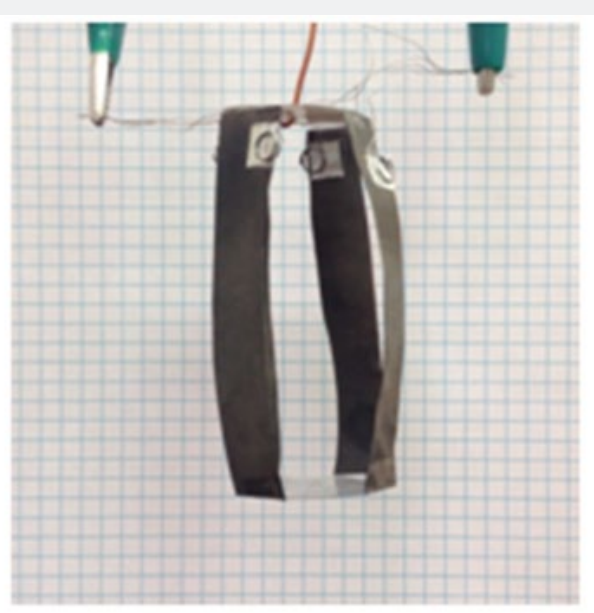

(a)

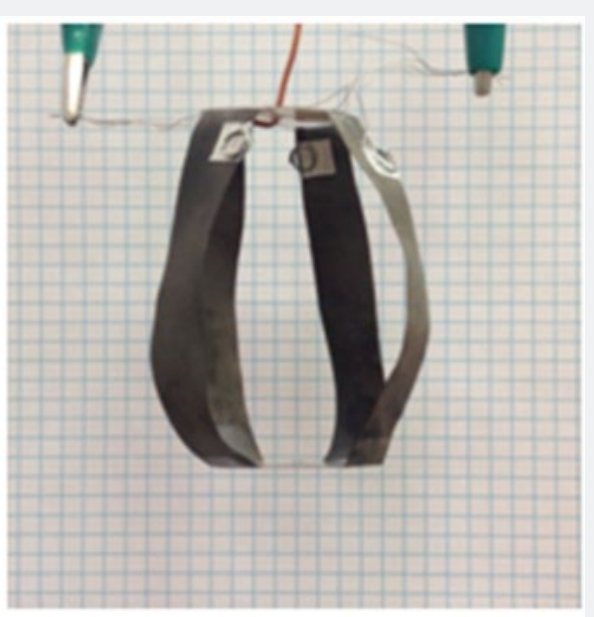

(b)

Figure 9: (a) A slit-tubular-actuator under-10 volts

(b)The same actuator under 10 volts. 


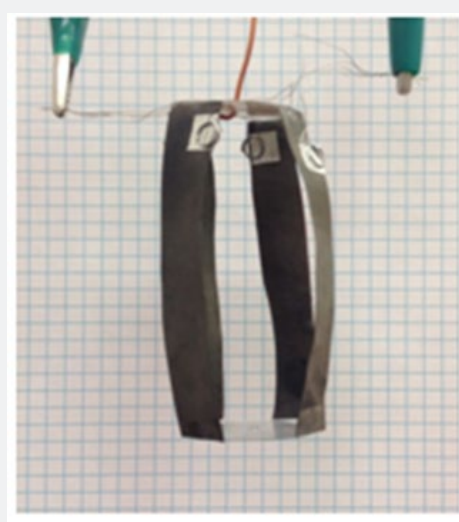

(a)

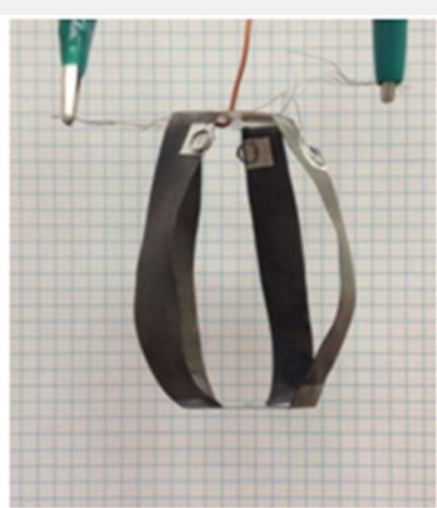

(b)

Figure 10: (a) A slit-tubular-actuator under -15 volts,

(b) The same actuator under 15 volts.

Some variations of these slit cylindrical configurations have and Shahinpoor $[23,24]$ in connection with jellyfish swimming been introduced previously by Yeom and Oh [30], Kamamichi [31] and multi-fingered soft robotic grippers, Figure 11.

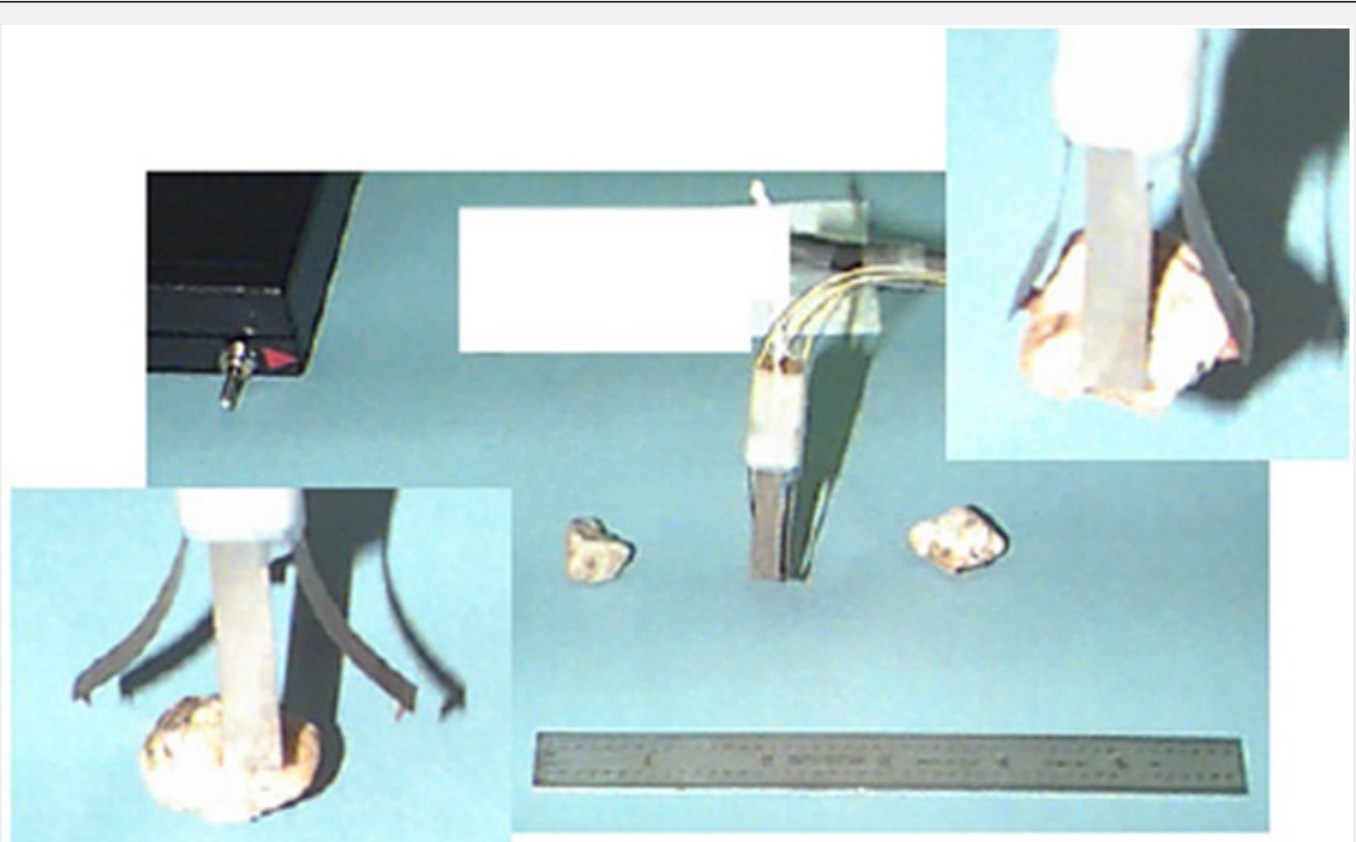

Figure 11: IPMC actuators as a soft multi-fingered robotic hand [12].

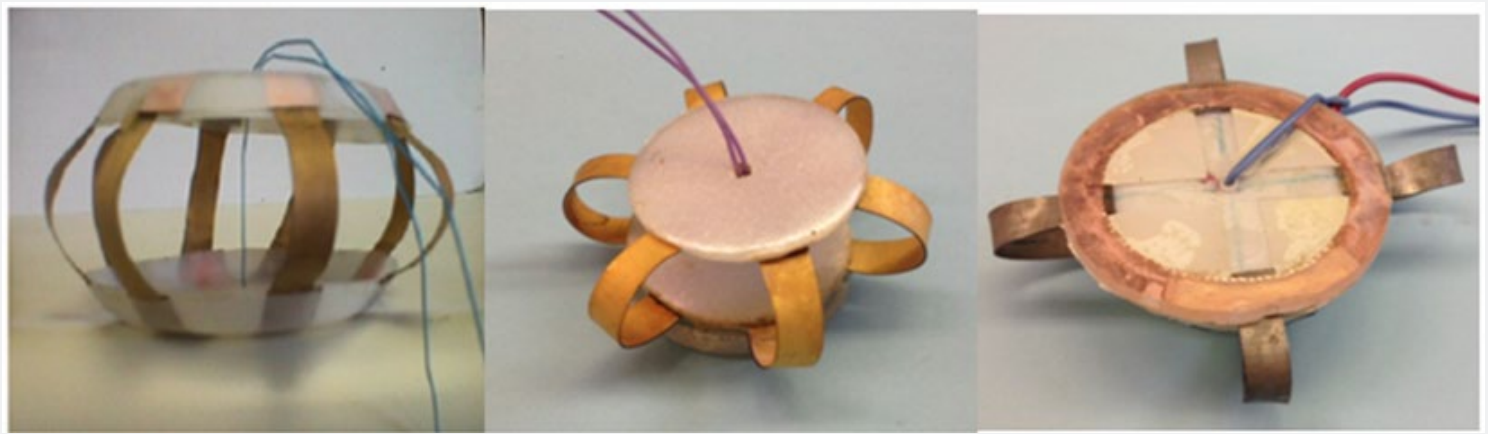

Figure 12: Various views of a cylindrical form of an IPMC actuator in clamped - clamped boundary conditions and configurations [13]. 


\section{Robotics \& Automation Engineering Journal}

In case of attaching the platform to an arm, these legs can act like multi-fingered soft biomimetic robotic grippers to grasp and manipulate objects.Other configurationswere also discussed by Shahinpoor and Kwang [13], as shown in Figure12.

The hinged-hinged configuration will help this possibility in a more efficient way to have longer actuation range.The bending for sensing is generally outward rather than inward because the slit IPMC strips considered are initially slightly bent outward to prevent any buckling instability.

From another point of views, slits on cylindrical configuration can be at an angle to introduce the helically twist and sloped version of helical form of IPMC shown in Figures 13(a\&b). This configuration of helically slit IPMC strips will generate axial and twisting motion upon actuation and yet it produces electricity once axially squeezed. Here since the twisting and retracting are related to each other, the model is considered to be more like an actuator.
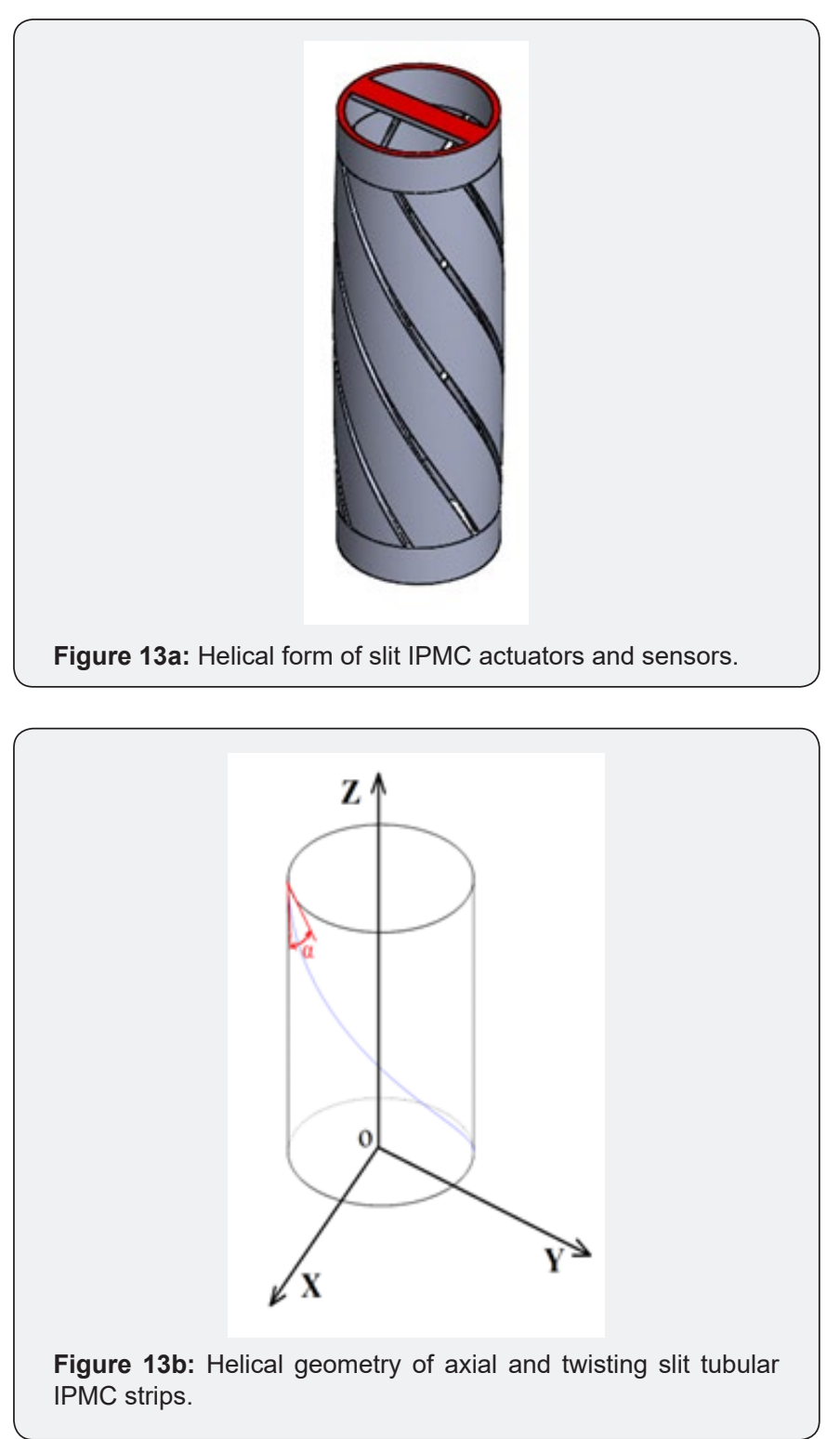

These configurations of slit IPMC strips produces twisting and linearly moving actuators similar to an automated drill/screw driver. Note that the helical IPMC actuator/sensor slit strips are like space curves with coordinates $\mathrm{x}, \mathrm{y}$ and $\mathrm{z}$ such that $x=r \cos \varphi$, $y=r \sin \varphi$ and $z=c \varphi$ for $\varphi \epsilon[0,2 \pi]$ where $\mathrm{r}$ is the radius of the helix and $\mathrm{c}$ is a constant giving the vertical separation of the helix's loops and $\varphi$ is an independent parameter. The tan $\alpha=\mathrm{c} / \mathrm{r}$ is slop of the strip sample. The curvature of the helix is given by $\kappa=r /\left(r^{2}+c^{2}\right)$, arc length is given by $s=\varphi\left(r^{2}+c^{2}\right)^{0.5}$, the torsion of a helix is given by $\tau=c /\left(r^{2}+c^{2}\right)$ such that $\kappa / \tau=r / c$.

Some studies and modeling for the types of combined linear/ twisting actuators reported in this paper have been proposed by Li and Kim [32] and Moeinkhah and Rezaeepazhand [33] and others. Figure 14(a,b\&c) depictvarious configurations of helical assembly of slit tubular IPMCs for actuation, energy harvesting and sensing with more geometric complexitiesare currently under investigations and will be reported later.
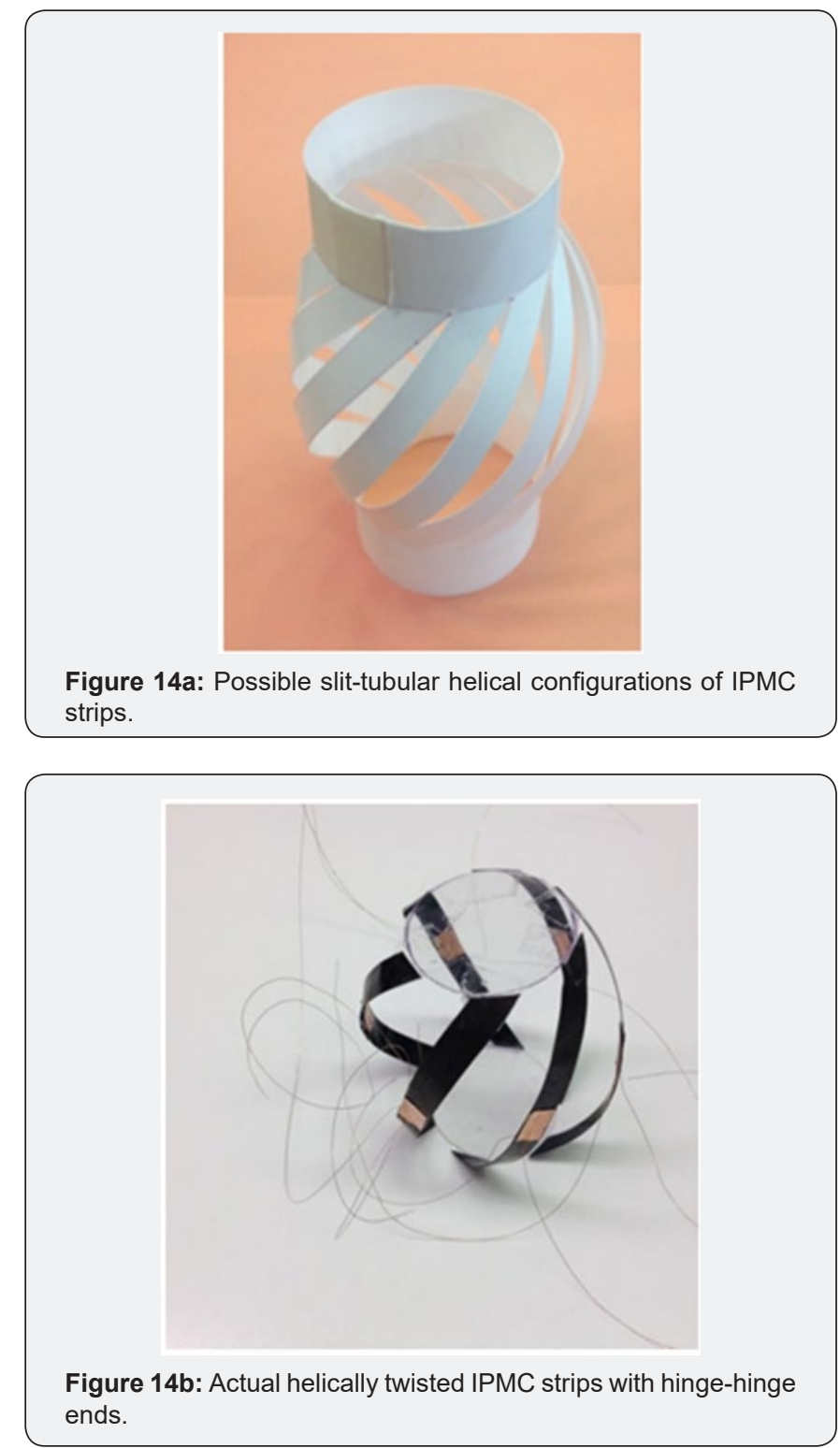


\section{Robotics \& Automation Engineering Journal}

\section{Soft Robotics Snake-Like Configurations Based on Linear-Torsional IPMC Actuators/Sensors}

Here the initial form of the device considered for the tubularactuators/sensors is a combination of axial and rotary movements that resembles slithering snakes,as shown in Figure 15 below:

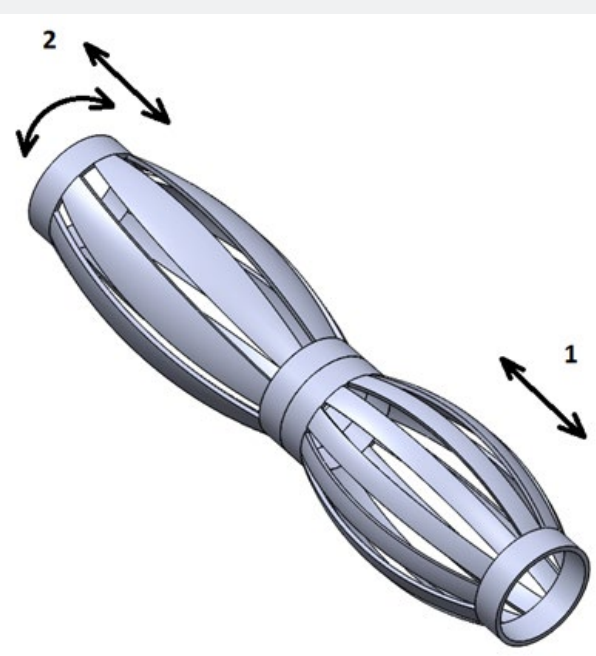

Figure 15: Possible undulating soft robotic arm made with slit tubular IPMC strips capable of bending, twisting, expansion, contraction and undulation.

In Figure 15 note that by a combination of movements 1 and 2, the device is capable of 3-D undulating anobject like an elephant trunk or a slithering snake-like flexible robotic manipulator [30]. Referring to Figure 16, in case of having more combination of these actuations configuration, possibility of having a more complex robot can be expected as it is depicted in Figure 16.

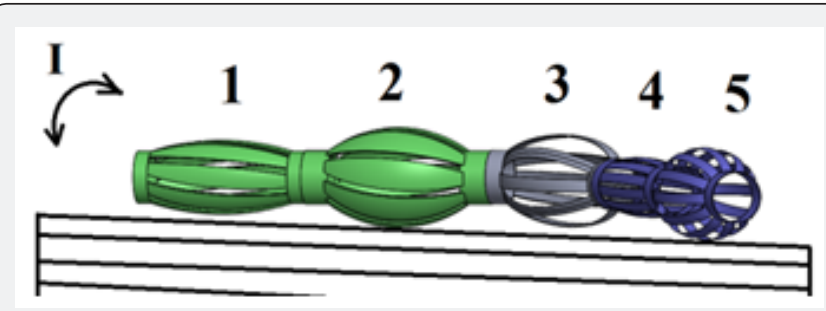

Figure 16a: Worm/snake/elephant trunk soft robot made of tubular IMPC actuators.

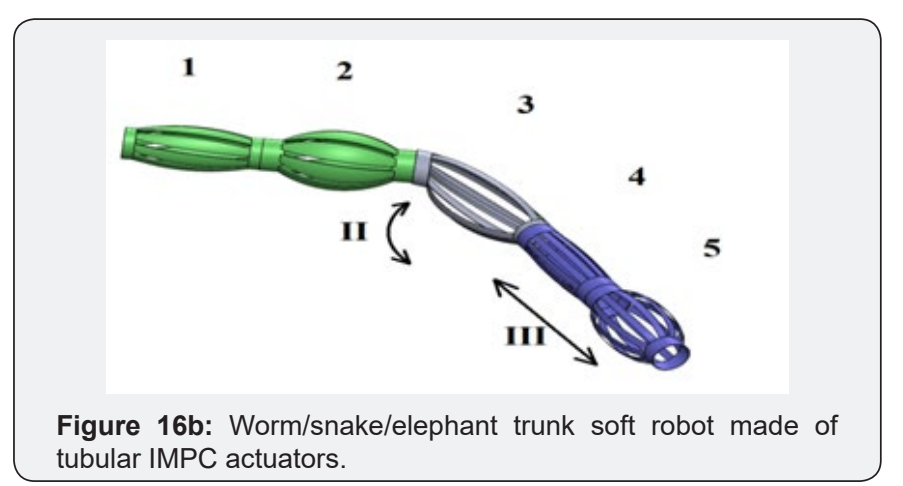

In Figure16 (a\&b) a variety of control and boundary condition can be applied to the assembly to move the soft snake-like, wormlike or elephant trunk-like soft robots. For the rotary movement in the direction denoted by I, actuators 1 and 2 are helically arrangedand can produce a combined rotation and slithering movement. The actuator number 3 in Figure 16b enables bending and twisting due to having unsymmetrically applied voltages to generatehelical and planar movements in the direction denoted by II. Yet extensional movements can be achieved by actuator 3 in Figure 16b in the direction denoted by III. Thus, we conclude that combination of cylindrically and helically arranged IPMC actuators are capable of generating elephant-trunk like motion.

\section{Conclusion}

A new family of IPMC linear actuators and sensors in the form of a slit tubular structure was introduced and discussed. These groups of actuator and sensors were considered to be made with bending/twisting slitIPMC elements symmetrically distributed around a cylindrical/tubular mantle. These types of soft actuators and sensors are capable of generating a combination of actuation, energy harvesting and sensing consisting of linear expansion, contraction, bending, twisting and rotating action similar to a slithering snake or an elephant trunk.Theslit bending elements around the tubular mantle were designed to be straight and parallel with the longitudinal axis of the cylinder for pure linear actuation and sensing in terms of the length of the entire cylinder. However, the possibility of helically winding the bending elements around the cylindrical mantle to produce a combination of linear and torsional actuation and bending for the cylinder was also briefly discussed. This introduction of linear/torsional actuation/energy harvesting and sensing opens the possibility for introducing new family of soft biomimetic robotic actuators and sensors. The next steps in development of advanced elephant-trunk-like soft biomimetic robotic actuators and sensors are to further explore various cylindrically and helically arranged IPMC strips. Currently, we are further exploring such possibilities towards fabrication an elephant-trunk type soft robot.

\section{References}

1. Shahinpoor M, Bar-Cohen Y, Simpson J0, Smith J (1998) Int J Smart Materials and Structures. 7(6): R15-R30.

2. Shahinpoor M, Bar-Cohen Y, Xue T, Simpson JO, Smith J (1998) Proceedings of SPIE's 5th Annual International Symposium on Smart Structures and Materials. San Diego, California, pp. 3324-3327.

3. Osada Y, Okuzaki H, Hori H (1992) A polymer gel with electrically driven motility. Nature 355: 242-244.

4. Oguro K, Kawami Y, Takenaka H, Trans J (1992) Micro-Machine Society. 5: 27-30.

5. Segalman DJ, Witkowski WR, Adolf DB, Shahinpoor M (1992) Int. Journal of Smart Material and Structures 1: 95-100.

6. Shahinpoor M (1992) Int. Journal of Smart Material and Structures 1: 91-94.

7. Doi M, Marsumoto M, Hirose Y (1992) Macromolecules 25: 5504-5511. 


\section{Robotics \& Automation Engineering Journal}

8. Oguro K, Asaka K, Takenaka H (1993) In Proceedings of the 4th International Symposium of Micro Machines and Human Science, Nagoya, pp. 38-40.

9. Adolf D, Shahinpoor M, Segalman D, Witkowski W (1993)US Patent Office, US Patent No. 5,250,167.

10. Oguro K, Kawami Y, Takenaka H (1993) US Patent Office, US Patent No. $5,268,082$.

11. ShahinpoorV M, Kim KJ (2001) Smart Materials and Structures Int J 10(4): 819-833.

12. Shahinpoor M, Kim KJ, Mojarrad M (2007) Artificial Muscles: Applications of Advanced Polymeric Nano Composites, CRC Press, Taylor \& Francis Publishers, London SW15 2NU, Great Britain, $1^{\text {st }}$ Edition.

13. Kim KJ, Shahinpoor M (2003) Smart Materials and Structures (SMS), Institute of Physics Publication 12(1): 65-79.

14. Kim KJ, Shahinpoor M (2002) Polymer. 43(3): 797-802.

15. De Gennes PG, Okumura K, Shahinpoor M, Kim KJ (2000) Europhysics Letters. 50(4): 513-518.

16. Asaka K, Oguro KJ (2000) Journal of Electroanalytical Chemistry 480:186-198

17. Shahinpoor M (2003) Electrochimica Acta 48(14-16): 2343-2353.

18. Shahinpoor M, Kim KJ (2002) Actuators and Sensors A. Physical, 96 (2/3) A, 3163, pp. 125-132.

19. Shahinpoor M, Kim KJ (2002) Applied Physics Letters (APL)80(18): 3445-3447.

20. Bahramzadeh Y, Shahinpoor M (2011) Proceedings of SPIE $18^{\text {th }}$ Annual International Symposium on Smart Structures and Materials, San Diego, California.

21. Bahramzadeh Y, Shahinpoor M (2011) Smart Materials and Structures Journal. 20(9): p. 7.

22. Shahinpoor M (2011) Bioinspiration and Biomimetics. Institute of Physics (IOP) Publishing Ltd., London, UK, 6: 1-11.

23. Shahinpoor M (2016) Ionic Polymer Metal Composites (IPMCs): Smart Multi-Functional Materials and Artificial Muscles. Volume II, Royal Society of Chemistry Publishers, Dr. Cara Sutton, MRSC, Commissioning
Editor, Thomas Graham House, Science Park, Milton Road, Cambridge CB4 OWF, UK.

24. Shahinpoor M (2016) Ionic Polymer Metal Composites (IPMCs): Smart Multi-Functional Materials and Artificial Muscles. Volume I, Royal Society of Chemistry Publishers, Dr. Cara Sutton, MRSC, Commissioning Editor, Thomas Graham House, Science Park, Milton Road, Cambridge CB4 OWF, UK.

25. Giacomello A, Maurizio Porfiri (2011) Underwater energy harvesting from a heavy flag hosting ionic polymer metal composites. Journal of Applied Physics 109(8): 084903.

26. Aureli M, Chekema Prince, Porfiri M, Peterson DS (2009) Energy harvesting from base excitation of ionic polymer metal composites in fluid environments. Smart materials and Structures 19(1): 015003.

27. Seidi M, Hajiaghamemar M, Tabatabaie E, Shahinpoor M (2015) Ionic Polymer-Metal Composites (IPMCs) as Impact Sensors. ASME 2015 Conference on Smart Materials, Adaptive Structures and Intelligent Systems. American Society of Mechanical Engineers.

28. Yamakita M, Kaneda Y, Asakaet K, Zhi-Wei Luo (2004) IPMC linear actuator with re-doping capability and its application to biped walking robot. IFAC Proceedings 37(14): 353-358.

29. Fallahi A, Bahramzadeh Y, Tabatabaie SE, Shahinpoor M (2017) A novel multifunctional soft robotic transducer made with poly (ethylene-comethacrylic acid) ionomer metal nanocomposite. International Journal of Intelligent Robotics and Applications 1(2): 143-156.

30. Yeom, Sung-Weon, Il-Kwon Oh (2009) A biomimetic jellyfish robot based on ionic polymer metal composite actuators. Smart materials and structures 18(8): 085002.

31. Kamamichi N, Yamakita M, Asaka K, Zhi-Wei Luo (2006) A snakelike swimming robot using IPMC actuator/sensor. Robotics and Automation, 2006. ICRA 2006. Proceedings 2006 IEEE International Conference on. IEEE.

32. Li Song-Lin, Kim WY, Cheng TH, Il-Kwon Oh (2011) A helical ionic polymer-metal composite actuator for radius control of biomedical active stents. Smart Materials and Structures 20(3).

33. Moeinkhah, Hossein, et al. (2015) Accurate dynamic modeling of helical ionic polymer-metal composite actuator based on intrinsic equations. IEEE/ASME Transactions on Mechatronics 20(4): 16801688.

\section{Your next submission with Juniper Publishers will reach you the below assets}

- Quality Editorial service

- Swift Peer Review

- Reprints availability

- E-prints Service

- Manuscript Podcast for convenient understanding

- Global attainment for your research

- Manuscript accessibility in different formats

( Pdf, E-pub, Full Text, Audio)

- Unceasing customer service

Track the below URL for one-step submission https://juniperpublishers.com/online-submission.php 